\title{
Invasive management needed for failed pyeloplasty?
}

Recent data suggest that more invasive and definitive techniques, such as redo pyeloplasty and ureterocalicostomy, are more successful than minimally invasive procedures for treating failed pyeloplasty in children and should be offered earlier in the follow-up assessment of these patients.

Romao et al. performed a retrospective record review of 455 children undergoing pyeloplasty from 2000-2010 at a single tertiary-care centre and found that the overall pyeloplasty failure rate was $5.9 \%$. The main indications for reintervention were worsening asymptomatic hydronephrosis (59\%), pain (26\%), and urosepsis (7.5\%).
Interestingly, age and indication for pyeloplasty had no impact on failure rates. "Failed pyeloplasty in children is rare, but, when it occurs, the mainstay treatment option is redo surgery," says Walid Farhat, who led the study. "We could not find any predictors of failure; thus, the only way to identify the failure is follow-up ultrasonography or renal scan."

$30 \%$ of patients improved after just one reintervention, $52 \%$ underwent two reinterventions, and $18 \%$ required three. The success rates of these reinterventions varied considerably, depending on the intervention modality. Double J stent insertion, endopyelotomy, redo

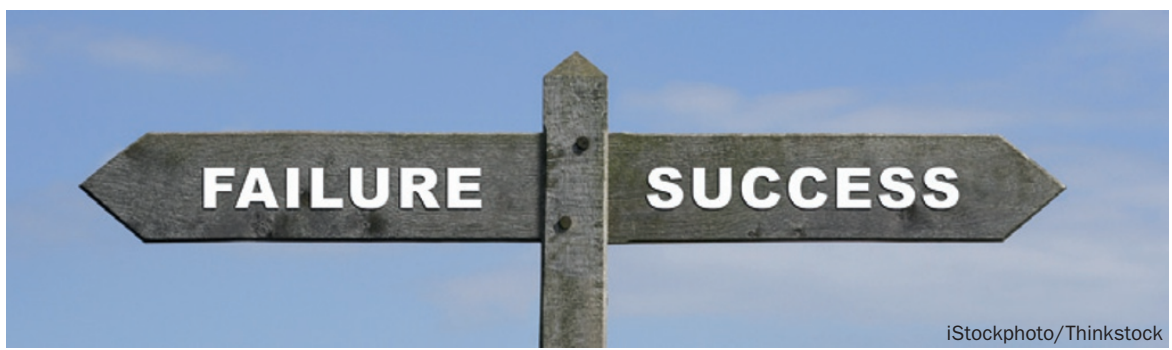

pyeloplasty, and ureterocalicostomy were associated with success rates of $6 \%, 18 \%$, $92 \%$, and $100 \%$, respectively, suggesting that more invasive procedures are more effective for correcting failed pyeloplasty.

However, in an accompanying editorial comment, Hal Craig Scherz suggests that delays to first and repeat interventions could explain the low success rates for minimally invasive procedures, as fixed fibrosis incurred during this time would almost certainly require open surgery.

"Some form of standardization to define pyeloplasty failure, such as a sonographic tool, would be helpful," responded Rodrigo Romao to Nature Reviews Urology. "One that is now undergoing validation is the Percent Improvement in AP Diameter. Multicentre prospective data for different treatment modalities are also needed."

Melanie Clyne

Original article Romao, R. L. et al. Failed pyeloplasty in children: revisiting the unknown. Urology doi:10.1016/ j.urology.2013.06.049 